\title{
Examining Stakeholder's Views On Refugee Healthcare Needs, Current Barriers in Accessing Healthcare Services and Future Healthcare Direction in New Zealand: A Qualitative Phenomenological Approach
}

\author{
Bafreen Sherif \\ Waitemata District Health Board \\ Ahmed Awaisu \\ Qatar University \\ Nadir Kheir ( $\square$ n.kheir@ajman.ac.ae ) \\ Ajman University
}

\section{Research Article}

Keywords: Refugees, New Zealand healthcare system, healthcare access, barriers

Posted Date: September 8th, 2021

DOI: https://doi.org/10.21203/rs.3.rs-877910/v1

License: (1) (i) This work is licensed under a Creative Commons Attribution 4.0 International License. Read Full License 


\section{Abstract \\ Background}

The annual New Zealand refugee quota was increased to 1500 places from 2020 onwards as a response to the global refugee crisis. The specific healthcare needs of refugees are not clearly understood globally and communication between healthcare providers and refugees remains poor.

\section{Methods}

A phenomenological qualitative methodology was employed to conduct semi-structured interviews among purposively selected stakeholders who work in refugee organisations and relevant bodies in New Zealand.

\section{Results}

The participants indicated the need for a national framework of inclusion, mandating cultural competency training for frontline healthcare and non-healthcare personnel, creation of a national interpretation phone line, and establishing health navigators. Barriers to accessing health services identified included some social determinants of health such as housing and community environment; health-seeking behaviour and health literacy; and social support networks. Future healthcare delivery should focus on capacity building of existing services, including co-design processes, increased funding for refugee-specific health services, and whole government approach.

\section{Conclusion}

Policymakers and refugee organisations and their frontline personnel should seek to address the deficiencies identified in order to provide equitable, timely and cost-effective healthcare services for refugees in New Zealand.

\section{Background}

Refugees and asylum seekers are a heterogeneous cohort varying widely in education, health literacy, cultural beliefs, and behaviors [2-3]. The dynamic process of displacement of population subgroups and its added pressure on the health and social structures of the receiving state, region or country, remains widely debated. While migration itself is not a risk factor for poor health outcomes as migrants are often comparatively healthy, vulnerability to physical, mental and social health problems may arise from the process and specific circumstances of migration, giving rise to public health concerns $[4-5,12]$. 
The specific healthcare needs of refugees are not clearly understood globally and communication between healthcare providers and refugees remains poor. Additionally, there is often inadequate responsiveness of healthcare systems due to poor preparation, further amplified by legal issues that refugees have to face with respect to access to health services. Approaches to managing refugee health problems or barriers to accessing health services have not sufficiently matched the pace of increasing challenges associated with the scale, diversity and disparity of current migration patterns $[9,12-13]$.

In 2014, the New Zealand Migrant Settlement and Integration Strategy was approved by Cabinet on behalf of the Ministry of Business, Innovation and Employment (MBIE) and in it, the New Zealand government's approach in settling and integrating recently arrived migrants had been outlined [10]. This research stems from the realization that there is a paucity of data on the health status of refugees in New Zealand; and postulates that the current healthcare provision to refugees and their families is fragmented and may only be marginally addressing healthcare disparities and needs in this population [1]. Therefore, the overall goal of this study is to explore the perspectives of key stakeholders on current refugee healthcare needs (including needs associated with pharmaceutical services), existing barriers faces by refugees in accessing and understanding healthcare services in New Zealand, and how these will contribute to the identification of future healthcare direction.

\section{Study Objectives}

This study aimed to: (1) explore the perceptions, attitudes, beliefs, and opinions of key stakeholders on refugee healthcare needs; (2) understand the existing barriers faced by refugees, as perceived by the relevant stakeholders, in accessing and understanding health care services in New Zealand and; (3) gauge future refugee healthcare directions so that existing understanding of this topic is developed and broadened.

\section{Theoretical/ conceptual framework}

Qualitative research was utilised as it explores the stakeholders' attitudes, values, beliefs, and opinions more fully [7]. An interview/topic guide was developed by the research team and included open-ended, neutral questions covering three general domains comprising current refugee healthcare needs, existing barriers to health access, and future healthcare delivery. The topic guide was reviewed and validated by the primary investigator (NK).

Researcher reflexivity was attained through the following measures; (1) maintaining a research diary, (2) acknowledging the researcher's existing feelings and experience with the phenomenon in question, and (3) explaining the influence of this experience on the data collection and analysis or findings and interpretation [12]. Trustworthiness that seek to ensure quality and credibility in qualitative research were addressed by using Lincoln and Guba's criteria (1985) and by implementing peer review processes by the research team, allowing for a member checking step and implementing thematic analysis [12]. Describing the research methodology in full, conducting a peer review, and maintaining a database of all research 
records and procedures achieved the dependability criterion. Additionally, participants were provided with a copy of the transcribed audio-recordings within a two-week turnaround period from the date of the interview so that they could have the opportunity to review and/or edit their transcripts. The confirmability criterion was attained through the maintenance of interview transcripts, describing the research procedures in full and utilising methods to enable future reproducibility and conducting a reflexivity analysis. Lastly transferability was ensured by recording and maintaining research-related outputs, and paying heed to the comprehensive presentation of data and discussion [12].

\section{Methods}

\section{Eligibility Criteria}

To be included in the study, the potential participant had to be 18 years or older, employed full-time for a minimum of one year with the respective refugee organisation, able to speak, read, and understand English, and must have had direct contact with refugees. Any employee who did not fulfil these inclusion criteria was not eligible to participate.

\section{Participants and Sampling}

Participants in this qualitative study were employees of organisations and services that represent refugees in New Zealand. To invite expression of interest (to join the study), managers of the respective refugee organisations were contacted by E-mail, and Participant Information Sheet (PIS) containing full information about the study, its aim and procedures, was provided. An informed consent form was also enclosed. Organisations agreeing for their employees to participate in the study were asked to display the study advertisements on noticeboards within the organisation and/or circulate the advertisement in an enewsletter format. Employees interested to join the study contacted the primary researcher and were provided with a study participant's pack containing the PIS that outlined the key information relevant to the study and the consent form to be returned to the primary investigator.

\section{Data Collection}

A semi-structured face-to-face, phone interview or Skype/Zoom interview approach, at the discretion of the participant, was used for data collection. The same interview question root as shown in Table 1, was utilized across the study participants allowing the elucidation of thoughts, opinions and perceptions of the stakeholders in keeping with an inductive thematic analysis methodology. Interviews were stopped when theoretical saturation was reached, at the conclusion of the eighteenth interview. The 18 interviews were conducted with individuals of diverse demographics (across gender, age and occupations) and from several refugee organisations across the different resettlement centres in New Zealand allowing for a diversity and breadth of data generation and results reflective of refugee stakeholders' views. Table 2 summarizes the characteristics of the interviewees. All the interviews were conducted over a four-month period (from September to December 2018) and each individual was interviewed once, with interviews averaging from 30-40 minutes in duration. Standards for reporting qualitative research (SRQR) was 
utilised to improve the quality of reporting of this qualitative research study [7]. All data was analysed using NViVO, version 12.0. 
Table 1

Interview Structure

\section{Focus Key Questions}

Refugee healthcare needs

-Which healthcare professionals do refugees see most frequently?

- How do refugees gain an understanding of health services?

-What are some common health-related issues that refugees experience?

- How supportive and responsive are health care delivery systems are towards refugee healthcare needs?

- How accepting do you believe we are of refugee healthcare choices (e.g. the use of traditional healers/medicine)?

- To what extent does the refugee experience in the country of origin affect the understanding of healthcare services on arrival?

Current barriers in accessing healthcare services
- Is there a reliance on accident and emergency services even where nonemergency treatment was appropriate?

-What are some challenges faced by refugees in accessing primary healthcare in NZ?

- How can we improve access to medicines and pharmacists?

- To what extent do refugees under or over-report symptoms of ill health?

- Do you think our health care system is adequately resourced to care for refugee patients and their families?

-What in your opinion is a barrier to refugees attending a healthcare service?

- Are there certain groups of refugees who are less likely to feel settled and/or have intrinsic barriers towards access to health?

- To what extent do you believe our services are 'culturally competent'? How does this translate across to refugee access to healthcare services?

- Do you believe there is continuity of care across the different healthcare services? 
Focus

Future healthcare direction

\section{Key Questions}

- Do you believe our healthcare services are responding sufficiently to perceived or expressed needs?

- How do refugees feel about the quality of the services that they access?

- How much influence do refugees believe they have in deciding the future direction of the services that they access?

-What channels are there for refugees to provide feedback, and/or make suggestions about the direction of healthcare services?

- Are healthcare services responding with enough momentum to refugee healthcare needs?

- Do you foresee a role for pharmacists at the heart of health services that care for refugees?

- How can pharmacists become involved in the refugee healthcare journey?

-What do you believe are some barriers in refugees accessing pharmaceutical services?

- How well do you believe refugees are integrating into New Zealand society?

-What are your visions for the future direction of refugee healthcare in New Zealand? 
Table 2

Stakeholder's characteristics

\begin{tabular}{|ll|}
\hline $\mathbf{N}$ & $\mathbf{1 8}$ \\
\hline Sex (female), $\mathrm{n}(\%)$ & $15(83.3)$ \\
\hline Age in years & 45.3 \\
\hline Occupational group & \\
\hline Nursing/Midwifery & 2 \\
\hline Physician & 6 \\
\hline Non-governmental organization & 3 \\
\hline Translation services & 1 \\
\hline Project Manager & 2 \\
\hline Clinical Psychologist & 1 \\
\hline Community Pharmacist & 2 \\
\hline Research Fellow & 1 \\
\hline Professional experience in refugee health in years & 3.7 \\
\hline
\end{tabular}

\section{Measures}

No identifying information relating to the refugee organisations appeared in the research reports or transcripts and instead, all the organisations with which participants worked were coded. The study protocol, study advertisement, PIS (managers and employees), consent forms (managers and employees), and the interview/topic guide were submitted to the University of Auckland Human Participants Ethics Committee (UAHPEC) for review and approval. This research was approved by the UAHPEC on 19/07/2018 for three years (approval reference number: 021308). A statement requesting the assurance of employers that participation or non-participation of their employees would not affect their relationship with their employees was included in both the manager and the employee PIS.

\section{Analysis}

An inductive methodology driven by the interviews was utilised and was based on a search for similarities and differences in data, which were then described in categories and higher-level themes. Given the nature of phenomenological research, participants' common and shared experiences were described through the use of individual interviews. All interviews were audio-recorded to enable transcribing and inductive analysis by the researchers. Transcribing followed an edited transcribing method, where emphasis was placed on understanding the content of the responses. To enhance rigor, the participants were offered an opportunity to review and edit their transcripts. Thematic content analysis was used to analyse the data generated from the interviews. This was operationalized through 
coding the edited transcripts using NVivo 12 for analysis, then generating categories from the coded terms or statements of interest in each transcript.

\section{Results}

\section{Thematic Analysis Results}

The key themes with their respective categories and supporting codes derived from the interviews across the three pre-determined domains as seen in Fig. 1. These pre- determined domains included: (1) refugee healthcare needs, (2) barriers to accessing healthcare services and, (3) future healthcare direction for refugees.

\section{Domain 1. Refugee Healthcare Needs}

\section{Theme 1: Overcoming Cultural and Linguistic Barriers Choice of Health Professionals to Visit}

Gender and cultural background of healthcare professionals was perceived to be an important consideration when accessing healthcare: "Some refugees prefer to go to a doctor from their background who speaks their language. Women, prefer women doctors." P1

\section{Healthcare Professional-Refugee Relationship}

The dynamics of the healthcare professional-refugee relationship are such that there is a high risk of staff burnout. One of the participants explained "Patients come to see a specific doctor not just for health needs but also for housing and employment - they trust the doctor more than just for health issues." P2

\section{Theme 2: Understanding Healthcare Service Delivery in New Zealand}

\section{Health Navigation Required for All Refugees}

Health navigators are required to guide and support the refugee community: "The first 6 months it's all so new and they're setting up schools, figuring out where to get food, where a bus stop is, and is their family safe? So it's often when life settles a bit then they think 'oh yeah, how do I do that'?" P3

\section{Communication}

A lack of understanding of health services and healthcare delivery systems may indirectly be demonstrated by potential or actual medication-related harm. This risk is of greater concern where language and health literacy barriers prevent the pharmacist from elucidating informed consent and understanding. As noted by one participant: "Being able to get them to understand the dosages, why we 
are asking this and maximum doses in a day - they've never had the healthcare, they've never had the range of medicines so you have to be careful not to make assumptions." P8

\section{Theme 3: Cultural Competency of Frontline Staff (Understanding Refugees and their Background)}

\section{Mechanisms in Healthcare System to Work with Culturally Diverse Individuals and Families}

A misunderstanding of refugees and their background on the part of service providers impedes healthcare access. One participant commented: "every year we run cultural competency training for frontline staff, but because of staff turnover we've still got challenges- the terminology is becoming blurred and people are not understanding who is a refugee, who is a migrant, who is an asylum seeker." P6

\section{Cultural Framework of Health (Collective vs. Individualistic Societies)}

Collective attitudes may clash with the western individualistic system and can be ethically challenging for health practitioners: "There are different cultural ways in which people might see a particular health condition or the way that the community might deal with that. Health professionals need to understand that people come from different worldviews." P12

\section{Theme 4: Patterns of Refugee Health Issues}

\section{Psycho-somatization of Illnesses}

There was a perceived element of somatization of psychological distress which healthcare professionals should remain vigilant of. " $A$ lot of people will have come from traumatic backgrounds and there may be a lot of psychological trauma that can manifest in a lot of pain in the body." P8

\section{Epidemiology of Global Chronic Diseases}

Patterns of refugee health issues have evolved over the last decade with a paradigm shift away from acute illnesses towards that of chronic diseases: "We are now dealing with chronic illness which we didn't see before. People were coming mostly from refugee camps where there were limited medical facilities and people just died... We have gone from a population of infectious diseases and deficiencies to a population with chronic disease."P5

\section{Theme 5: Healthcare System Responsiveness to Refugee Needs}

\section{Refugee Expectations of Healthcare System}


Refugees may have unrealistic expectations and perceptions of the capability of the New Zealand's health services particularly around the rules and regulations of medicines available over-the-counter without a prescription: "there is frustration at not being able to buy antibiotics and other medicines, the fact that GPs are gatekeepers and they're very restricted about giving out antibiotics." P6

\section{Social Determinants of Health}

There appears to still be perceived difficulties with societal acceptance of refugees, but this not overshadowed by the fact that as a country New Zealand is thought to be faring comparatively well on the global stage. This was illustrated by a participant who suggested that "most people feel welcomed and accepted but then people who are already disenfranchised and marginalized then go into communities that are in themselves disenfranchised and marginalized. There is that idea of a refugee coming in to take their houses, take their space-sadly they come and live in a world that propagates this idea of the working class or the poorest of the poor and 'why don't we look after our own first?"P3 Domain 2. Refugee Healthcare Barriers

\section{Theme 1: Worldviews and Health Literacy of Refugees}

\section{Healthcare Professional Training/Skills in Refugee Health}

Refugee healthcare is perceived to be a niche area: "within the refugee sector, the expertise and the experiences are quite good and getting better - outside of that, there is a lot of naivety within the society and community." P13

\section{Worldviews of Refugees/Pre-conceived Ideas about Health}

Refugees are not the only category of patients, but also new migrants who present to a hospital preferentially as this is the system of healthcare that they are accustomed to. An interviewee noted: "for a lot of countries where these people come from; if they're sick they just go to the hospital."P2

\section{Access to Medicines and to Pharmacists}

The diversity in the ethnic makeup of the pharmacist workforce is perceived as beneficial and other healthcare programs were advised to follow suit: "we have a much more diverse pharmacy workforce: it would be really good to get more students with former refugee backgrounds into health professional courses so that there is a range of different languages that's able to be spoken." P8

\section{Theme 2: Structure of the New Zealand Healthcare System Healthcare System Difficult for Refugees to Negotiate and Engage with}

Access to GP services for refugees is reasonable; however, primary care is perceived to be wider than the GP practice: "GP access is pretty good, but there is a whole range of other services and supports that 
mainstream people can access but unless somebody assists the refugee to that service they're probably not going to access it, and unless that service is equipped to meet their linguistic and cultural needs then they may not want to use it." $P 1$

\section{Receiving Timely Healthcare / Seamless Care}

Lack of transport and communication barriers are huge and limit refugee access to timely healthcare especially when refugees may require urgent healthcare and interpreters cannot be organised: "barriers to access include lack of interpreters, transport, location, how to get information to people once you're in the sessions."P3

\section{Domain 3. Future Healthcare Direction}

\section{Theme 1: Need for Further New Zealand-based Research Examining Refugee Health in the Future}

\section{Data Collection Methods in Refugee Health Research not Robust}

Services are engaged in consultations with refugee groups, but tangible changes are perceived as slow and this may relate data collection methods for refugee health: "there are endless consultations... it does really come down to how health stats are collected and reported... previously there was just one big Asian group, which statistically makes no sense. Now, many reports will have European, Maori, Pacific, and Asian and then other, but the MELAA group, if you're using that as a proxy for refugee, is often not reported." P1

\section{Theme 2: Empowerment of Refugees to Guide and Steer Future Healthcare Delivery}

\section{The 'Refugee Experience' is disempowering}

People from refugee backgrounds working within health services tend to feel frustrated with the lack of opportunities for improvement of these services. A lack of improvement is often attributed to the difficulties in catering for the diverse and non- homogeneous refugee cohort within the financial limitations of the system: "The sorts of things that come back are 'refugee groups are so small, we can't do anything, there's so many of them and they're so diverse that we might as well not try to do anything to accommodate cultural needs'" P1

'Refugee Voices' Difficult to Extract (Refugees are a Heterogeneous Population, no 'One- size Fits All' Model)

Often, refugees not working within the healthcare services are perceived to lack the realization that they have a 'voice' and can impact the future of services, which is an area that requires change: "I don't think 


\section{Theme 3: Achieving Equitable Targeted Health Outcomes for Refugees}

\section{Delivery of Health-related Information Requires On-going Planning and Review}

Pharmacists are noted to be frontline healthcare professionals and have a role as facilitators in the refugee health journey: "Pharmacists can deliver training and education sessions for refugees - arrange workshops, meetings, and information sessions to explain the role of pharmacist and also how pharmacists can be helpful to them in the community." P4

\section{Capacity Building/Streamlining Existing Services}

Healthcare services make efforts to respond to the needs of refugees but at times these needs fall outside the realm of expertise of staff and/or may occur too slowly for there to be quantifiable change: "some of the health conditions are quite unique to certain groups. For example, female genital mutilation (FGM) - it's practiced around the world in many countries and there are some nuances of these which we are not familiar with." P2

\section{Discussion}

Forced migration and the resultant creation of refugees is a top priority on the policy agendas of many of the world's leading member states of the World Health Organisation (WHO). In the New Zealand setting, there is a trend for many services to evolve reactively to the arrival of migrants and refugees and are adapted to the perceived or expressed needs of the population $[2-3,8]$

Clearly to address healthcare access barriers, there is a need for translation services in primary healthcare, information about the New Zealand health system and pertinent health information to be made available in ethnic minority languages [10-11]. This aspect has been emphasized multiple times by several participants this study. Cultural competency training for the healthcare workforce and capacity building in mainstream services are needed rather than establishing separate ethnic-specific services. Furthermore, there is a need to include refugee and migrant groups in both the national and regional health policy and strategy and to standardise ethnic data collection systems in a manner that allows the recognition of ethnic minority groups in New Zealand. There is also an on-going need to improve migrant and refugee research given the extensive gaps in research and information about refugee and migrant population health including a need for longitudinal data on the health of refugee/migrant populations in New Zealand. This study draws on national and international literature as a comparison. It is recognised and acknowledge that much of the refugee health research conducted in New Zealand has been on a 
small scale and locally- based. Therefore, the findings have limited generalizability to all refugee/migrant populations and all health issues [11].

Akin to the findings of Pavli et al. (2017), this research also noted that communication gaps between healthcare providers and refugees exist, creating difficulties in clearly elucidating the specific health needs of refugees $[6,9,13]$. Additionally, there is often an inadequate response of healthcare systems to these needs due to poor preparedness and/or fragmentation of care. Refugees are perceived to be a highneeds population group requiring more intensive and frequent support from healthcare professionals. Barriers to accessing healthcare services by refugees still exist, though efforts have been made in the larger centres such as Auckland to increase consultation times through refugee wrap-around services. Approaches to managing refugee health problems need to contend with the increasing challenges associated with healthcare delivery to a heterogeneous refugee population that is growing in size and displays a diversity and disparity of healthcare needs and unique barriers to access [10-14].

Effective healthcare provision may be impeded by the lack of healthcare professionals with the required experience of working with refugees. The potential role of primary care in reducing inequity of access and of raising the quality of care should be fully explored, and the use of healthcare navigators is recommended in advocating for refugees as is utilizing the role of community pharmacies as healthcare partners in assisting with individual refugee access, and in the delivery of community education about the New Zealand healthcare system.

This research supports collateral, multi-organisational efforts that pool existing resources in the most culturally appropriate manner under the umbrella of an overarching government-led national refugee framework with a focus on health equity. Refugees often have a collective attitude to healthcare and have a need for readily accessible interpreters, culturally competent healthcare practitioners and health information that is translated and made available in all the common refugee languages. Refugees want to feel welcomed, listened to and respected at all points of healthcare access.

\section{New Contribution to the Literature}

The findings of this study suggest a more proactive and refugee-responsive public health system as one where its national ethnicity system is able to epidemiologically categorise and identify refugees in a population. This research highlights the limitations around how New Zealand refugee health data is gathered and maintained, on a regional and national level, and recommends a review of refugee health information and ethnicity classification as part of the on-going scope for future refugee healthcare direction. Furthermore, there is a lack of a central data repository that compiles and maintains refugee health data obtained during the domestic health screenings which fragments care for refugees who may be enrolled with a GP but not necessarily heavily engaged with the GP services and favour attendance of emergency and Accident and Emergency services. Due to a lack of robust centralization of data, population-specific risk factor information is not easily recognised or retained. Therefore, policymakers need to strengthen the reporting process by creating a centralized system that recognises and retains 
refugee population-specific risk factor information relevant to the New Zealand and global context. We support the need for the creation of an overarching New Zealand-specific framework for addressing cultural diversity and for policy and funding strategies to recognise health needs in refugee groups.

\section{Conclusion}

Interviewees indicated the need for a national framework of inclusion, mandating cultural competency training of frontline personnel, the creation of a national interpretation phone line, and establishing health navigators. Barriers to accessing health services identified included social determinants of health such as housing and community environment; health seeking behaviour and health literacy; and social support networks. Future healthcare delivery should focus on capacity building of existing services, encompassing co-design processes, increased funding for refugee-specific health services, and harnessing a whole- government approach.

\section{Abbreviations}

FGM

female genital mutilation

SRQR

Standards for reporting qualitative research

\section{Declarations}

\section{Ethics approval and consent to participate}

This research was approved by the University of Auckland Human Participants Ethics Committee (UAHPEC) on 19/07/2018 for three years (approval reference number: 021308). A statement requesting the assurance of employers that participation or non-participation of their employees would not affect their relationship with their employees was included in both the manager and the employee PIS.

\section{Consent for publication}

The participants agreed to the publication of the study results. The anonymity of the participants and the confidentiality of the data were ensured.

\section{Availability of data and materials}

The datasets used and/or analysed during the current study are available from the corresponding author on reasonable request.

\section{Competing interests}

The authors declare that they have no competing interests. 


\section{Funding}

This research received a small post-graduate study grant of $\$ 500$ from the School of Pharmacy, Auckland.

\section{Authors' contributions}

Bafreen Sherif: contributed to conception and design of the study, undertook data collection and analysis, Nadir Kheir: contributed to conception and design of the study, undertook data collection and analysis. Ahmed Awaisu: contributed to conception and design of the study. All authors contributed to data interpretation, writing- original draft, and approved the final manuscript for submission.

\section{Acknowledgements}

We acknowledge all participating stakeholders who gave so generously of their time and shared their experiences and views with our research team.

\section{References}

1. Babar, Zaheer-Ud-Din \& Scahill, Shane \& Pengelly, Kelly \& Garg, Sanjay \& Shaw, John. (2013). Migrant health in New Zealand: Exploring issues concerning "medicines access and use. Journal of Pharmaceutical Health Services Research. 4. 41-49. 10.1111/j.1759- 8893.2012.00105.x.

2. Blom, N., Huijts, T., \& Kraaykamp, G. (2016). Ethnic health inequalities in Europe. The moderating and amplifying role of healthcare system characteristics. Social Science \& Medicine, 158, 43-51. DOI: 10.1016/j.socscimed.2016.04.01

3. Bradby H, Humphris R, Newall D, Phillimore J. Public health aspects of migrant health: a review of the evidence on health status for refugees and asylum seekers in the European Region. Copenhagen: WHO Regional Office for Europe; 2015 (Health Evidence Network synthesis report 44).

4. Chase LE, Cleveland J, Beatson J, Rousseau C. The gap between entitlement and access to healthcare: An analysis of "candidacy" in the help-seeking trajectories of asylum seekers in Montreal. Social Science \& Medicine (1982). 2017 Jun;182:52-59. DOI: 10.1016/j.socscimed.2017.03.038.

5. Heeren, Martina; Wittmann, Lutz; Ehlert, Ulrike; Schnyder, Ulrich; Maier, Thomas; Müller, Julia (2014). Psychopathology and resident status - Comparing asylum seekers, refugees, illegal migrants, labor migrants, and residents. Comprehensive psychiatry, 55(4):818-825. DOI: https://doi.org/10.1016/j.comppsych.2014.02.003

6. Johnson, D. R., Ziersch, A. M., \& Burgess, T. (2008). I don't think general practice should be the front line: Experiences of general practitioners working with refugees in South Australia. Australia and New Zealand Health Policy, 5(20), 20. doi: 10.1186/1743-8462-5-20

7. Kaae, S., \& Traulsen, J. M. (2015). Qualitative methods in pharmacy practice research. In Z- U-D. Babar (Ed.), Pharmacy Practice Research Methods (pp. 49-68). Switzerland: Springer. https://doi.org/10.1007/978-3-319-14672-0_4 
8. Khan, M. S., Osei-Kofi, A., Omar, A., Kirkbride, H., Kessel, A., Abbara, A.,.. . Dar, O. (2016). Pathogens, prejudice, and politics: the role of the global health community in the European refugee crisis. doi: 10.1016/S1473-3099(16)30134-7

9. Lamb, Cathryn \& Smith, Mitchell. (2002). Problems refugees face when accessing health services. New South Wales public health bulletin. 13. 161-3

10. Ministry of Health. 2016. Pharmacy Action Plan: 2016 to 2020. Wellington: Ministry of Health. Online: 978-0-947515-14-0

11. Mortensen, A. (2011). Public Health System Responsiveness to Refugee Groups in New Zealand: Activation from the Bottom Up. Social Policy Journal of New Zealand/Te Puna Whakaaro, (37), 123.

12. Mukhalalati, B., \& Awaisu, A. (2019). Principles, paradigms, and application of qualitative research in pharmacy practice. Elsevier.

13. Pavli, D. A., \& Maltezou, D. H. (2017). Health problems of newly arrived migrants and refugees in Europe. Journal of Travel Medicine, 24(4). https://doi.org/10.1093/jtm/tax016

14. Saunders, H. (2015). Meeting the health needs of newly arrived refugees. The New Zealand Medical Journal, 128(1423), 89. 1423-16-october-2015/6693

\section{Figures}




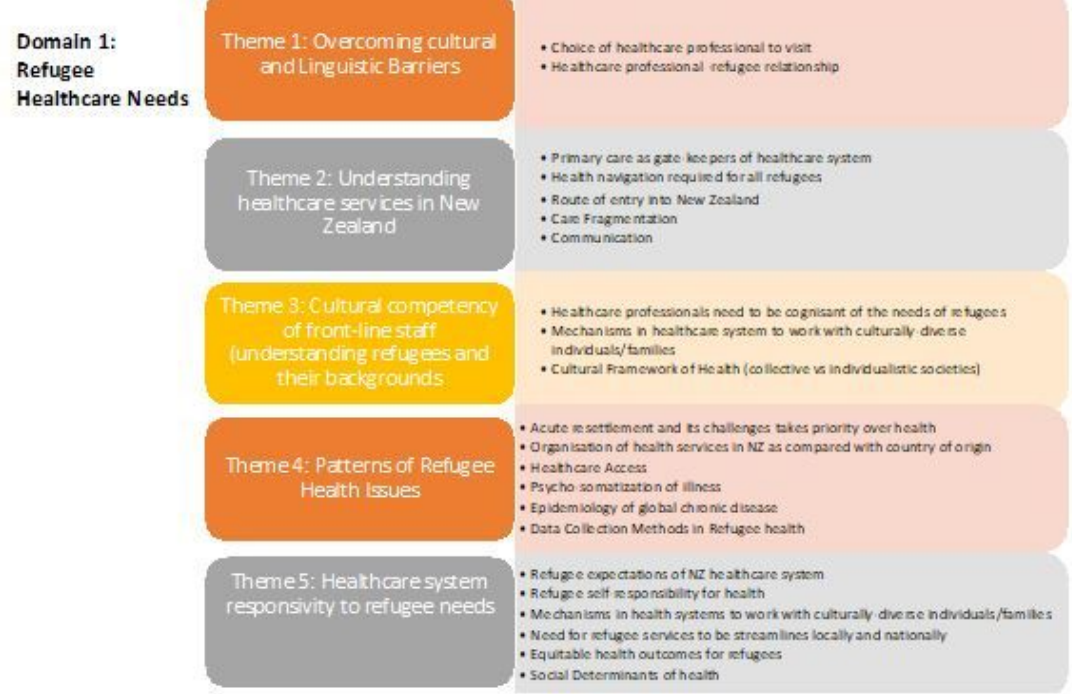

Domain 2:

Refugee

Healthcare

Barriers

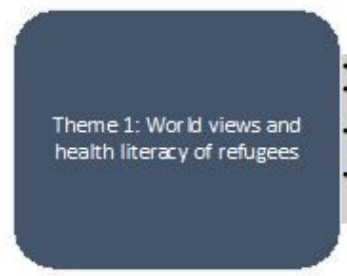

- Healthicare Professional expertise in Refugee Health - Presenting to Emergency Departunents and/or After-
hours Care Preferentially to General Practice

-Worldviews of Refugee/ Pre-conceived Ideas about

Health

- Access to Medicines and to Pharmacists

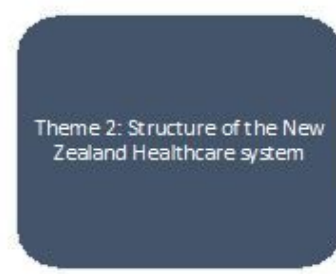

- System Difficult for Refugees to Negotiate and Engage

with

-Understanding and Engaging with Health-related

Information/Advice and Im plementing into Practice

- Cultural Competency of Frant-line Stafl

- Receiving Timely Healt hcare/ Seamless Care

-Working in a Health Partnership

Domain 3: Future

Healthcare

Direction
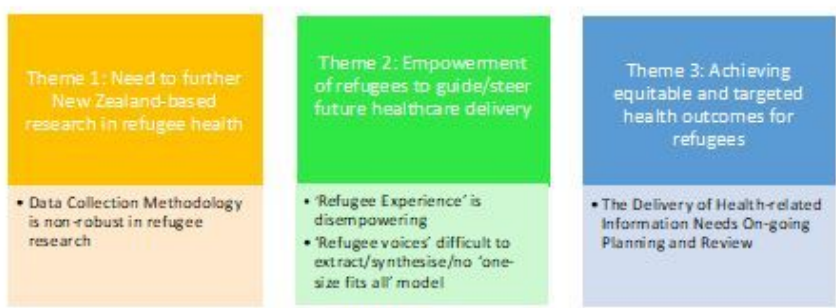

Figure 1

Summary of Key Themes Emerging from the Study Findings 